\title{
Immunosuppressive regimens for adult liver transplant recipients in real-life practice: consensus recommendations from an Italian Working Group
}

\author{
Umberto Cillo ${ }^{1}$ Luciano De Carlis ${ }^{2,3} \cdot$ Massimo Del Gaudio $^{4}$. Paolo De Simone ${ }^{5}$. Stefano Fagiuoli ${ }^{6}$. \\ Francesco Lupo $^{7}$. Giuseppe Tisone ${ }^{8} \cdot$ Riccardo Volpes $^{9}$
}

Received: 13 May 2020 / Accepted: 6 September 2020 / Published online: 24 October 2020

(C) The Author(s) 2020

\begin{abstract}
It is a well-recognized fact that implementing new guidelines in clinical practice may be difficult; therefore the Italian Society for Organ and Tissue Transplantation (SITO) set out to define practical immunosuppression tools for the management of liver transplantation patients. In 2017, an Italian Working Group of liver transplant experts and hepatologists issued a set of consensus statements along with evidence-based recommendations on the use of everolimus after liver transplantation. This article presents the evidence- and consensus-based algorithms developed within the Italian Working Group, which are aimed towards guiding clinicians in the selection of immunosuppressive regimens for the management of adult liver transplant recipients in real-life practice. The liver transplant recipient population, typically managed in clinical practice, was divided into the following categories: (1) standard patients; (2) critically ill patients; (3) patients with a specific etiology; (4) patients with hepatocellular carcinoma; (5) and patients with de novo malignancies. The algorithms are divided into two parts, according to the time from transplantation (0-3 months and > 3 months) and are discussed here along with relevant supporting literature, when available. Ultimately, it is hoped that the evidence- and consensus-based algorithms developed within the Italian Working Group, and presented here, contribute to simplify, personalize, and optimize immunosuppression of liver transplantation recipients in clinical practice.
\end{abstract}

Keywords Calcineurin inhibitor $\cdot$ Chronic kidney disease $\cdot$ Consensus recommendations $\cdot$ Everolimus $\cdot$ Hepatocellular carcinoma $\cdot$ Immunosuppression $\cdot \mathrm{mTOR}$ inhibitor $\cdot$ Sirolimus $\cdot$ Solid-organ transplantation $\cdot$ Tacrolimus

\section{Abbreviations}

ADPKD Autosomal dominant polycystic kidney disease

AILD Autoimmune liver disease

CCA Cholangiocarcinoma
CI

CNIs

eGFR

HCC

IQR
Confidence interval

Calcineurin inhibitors

Estimated glomerular filtration rate

Hepatocellular carcinoma

Interquartile range
Stefano Fagiuoli

sfagiuoli@asst-pg23.it

1 Hepatobiliary and Liver Transplant Unit, University Hospital of Padua, Padua, Italy

2 Department of General Surgery and Transplantation, Niguarda Hospital, Milan, Italy

3 School of Medicine, University of Milano-Bicocca, Milan, Italy

4 Department of General Surgery and Transplantation, Policlinico S. Orsola-Malpighi, Bologna, Italy

5 Hepatobiliary Surgery and Liver Transplantation Unit, University of Pisa Medical School Hospital, Pisa, Italy
6 Gastroenterology Hepatology and Transplantation, Papa Giovanni XXIII Hospital, Piazza OMS, 124127 Bergamo, Italy

7 Department of General Surgery, Azienda Ospedaliera Città Della Salute E Della Scienza, Turin, Italy

8 Department of Experimental Medicine and Surgery, Transplantation Surgery, Policlinico Tor Vergata University of Rome, Rome, Italy

9 Mediterranean Institute for Transplantation and Advanced Specialized Therapies (ISMETT/IRCCS), Palermo, Italy 


$\begin{array}{ll}\text { MDRD } & \text { Modification of Diet in Renal Disease } \\ \text { MELD } & \text { Model for End-Stage Liver Disease } \\ \text { metaCRC } & \text { Metastatic colorectal cancer } \\ \text { NET } & \text { Neuroendocrine tumors } \\ \text { PLD } & \text { Polycystic liver disease } \\ \text { PCLD } & \text { Isolated polycystic liver disease } \\ \text { RR } & \text { Risk ratio } \\ \text { SITO } & \text { Italian Society for Organ and Tissue } \\ & \text { Transplantation }\end{array}$

\section{Introduction}

Despite considerable progress in solid organ transplantation leading to increased patient and graft survival, complications associated with maintenance immunosuppressive therapy required to prevent rejection remain a major issue [1]. In liver transplant recipients, for example, the chronic use of calcineurin inhibitors (CNIs) has been shown to increase the risk of renal dysfunction, metabolic disorders, neurotoxicity, and de novo malignancies [2-8]. As an example, approximately $20 \%$ of liver transplant recipients experience chronic renal failure within 5 years of transplantation [7].

Consequently, extensive efforts have been devoted to developing strategies for reducing or withdrawing CNIs [9]. In this regard, the mTOR inhibitors, sirolimus, and everolimus have attracted considerable attention because of their immunosuppressive and antiproliferative properties [1]. Furthermore, a large body of evidence has shown that mTOR inhibitor-facilitated CNI reduction is associated with nephroprotective effects in both de novo and maintenance liver transplant recipients [10-14].

In 2017, an Italian Working Group, composed of senior representatives from Italian liver transplant centers, issued a set of consensus statements with evidence-based recommendations on the use of everolimus after liver transplantation [15]. The statements addressed four areas of practical interest: (1) kidney function; (2) timing of everolimus introduction, reduction of CNI exposure, and risk of graft rejection; (3) antiproliferative effect of everolimus; and (4) management of everolimus-related adverse events [15]. According to these recommendations, strategies for the prevention of renal impairment should be implemented early following transplantation to obtain good outcomes (typically after 4 weeks); in addition, owing to its antiproliferative properties, everolimus is also recommended for the prevention of de novo malignancies and recurrence of hepatocellular carcinoma (HCC) [15].

However, the implementation of new recommendations is rarely a straight-forward process in real-life. Indeed, the multicenter, observational study SURF, which assessed the management of patients undergoing liver transplantation in Italy, has shown that most liver transplant recipients with chronic kidney disease are not managed according to current guidelines, with less than $20 \%$ being switched from standard CNI-based immunosuppressive regimens to renal-sparing alternatives [16]. In addition to the previously mentioned recommendations [15], a further objective of the Italian Working Group of transplantation experts was to identify practical issues not fully covered by the guidelines and to improve the implementation of these recommendations in clinical practice.

This article presents the evidence- and consensus-based algorithms developed within the Italian Working Group and aimed at guiding clinicians in the selection of immunosuppressive regimens for the management of various categories of adult liver transplant recipients.

\section{Methods}

This project was conceived under the auspices of the Italian Society for Organ and Tissue Transplantation (SITO) to define practical immunosuppression algorithms for the management of adult, ABO-compatible liver transplantation patients and was prompted by the awareness that implementing new guidelines in clinical practice may be difficult. It was launched in 2017 by a group of 8 Italian expert transplant physicians (scientific board), most of whom were among the authors of the recommendations for the use of everolimus in liver transplantation published in the same year in Transplantation [15]. The expertise of the scientific board members was substantiated by their publication records, participation in national/international scientific meetings and clinical trials, academic rank, and clinical experience in liver transplant surgery or transplant hepatology, as well as the use of mTOR inhibitors. With regard to everolimus use, it should be noted that in Italy it has been used off-label for liver transplantation since 2006, well before the EMA approval in this indication, thanks to specific legislation and based on evidence from phase II clinical trials. The aim of this project was to produce evidence- and consensus-based recommendations for immunosuppressive therapy in adult liver transplant recipients, with a focus on clinical practice.

In 2017, the scientific board convened in Milan to share and discuss the clinical experience with everolimus at their centers and to review the relevant literature, with the purpose of identifying unresolved issues and producing a standard protocol for immunosuppression, in line with current recommendations for everolimus use [15] and based on published evidence when available, clinical experience, and consensus. The scientific board used a three-step modified Delphi method for reaching consensus, as previously described [15].

In Step 1, the scientific board convened in Milan at a face-to-face meeting during which it was decided to divide the liver transplant recipient population, typically 
managed in clinical practice, in the following categories: (1) standard patients; (2) critically ill patients; (3) patients with a specific etiology; (4) patients with HCC; (5) and patients with de novo malignancies. ABO-incompatible liver transplant patients were excluded from the target population due to the scarcity of such practice in Italy. With respect to HCC patients, previous national consensus conferences suggested liver transplantation for T2 patients $(\geq 2 \mathrm{~cm})$, whilst the upper tumor size limit or stage was left to the discretion of each transplant center based on donor graft availability and transplant benefit [17]. The scientific board drafted immunosuppressive protocols for each patient category. These protocols were to be submitted for assessment to a panel of clinicians (consensus panel). The consensus panel was composed of clinicians from Italian liver transplant centers ( 21 centers were invited; 16 centers participated with a total of 31 representatives; names and affiliations of clinicians are listed in Appendix A).

In Step 2, the scientific board and consensus panel met in Rome at a plenary meeting. The members of the consensus panel were asked to express their agreement or disagreement on the treatment protocol proposed for each patient category using a Likert scale from 1 to 5 (1, strongly disagree; 2 , disagree; 3 , neutral; 4 agree; 5 strongly agree). The positive consensus was defined by $>70 \%$ agreement on a given protocol (expressed as the percentage of respondents scoring the protocol with either 4 or 5) [15]. A positive agreement was reached on all immunosuppression protocols.

The treatment algorithms presented here were finalized during a final face-to-face meeting of the scientific board held in Milan (Step 3). The algorithms have been available since September 2018 on the SITO website (https://www. societaitalianatrapiantidiorgano.com/).

After a period of real-world practice, the treatment algorithms were amended and approved by the consensus panel at a plenary meeting held in December 2019.

\section{Strategies for immunosuppression in liver transplant recipients}

The algorithms for immunosuppressive therapy in adult, ABO-compatible liver transplant recipients, according to the patient category, are shown in Figs. 1-5. All algorithms are divided into two parts, according to the time from transplantation: $0-3$ months and $>3$ months. General remarks on the algorithms are shown in Table 1 and additional comments concerning the different recommended immunosuppressive therapies are shown in Table 2. The algorithms are discussed in the following sections along with relevant supporting literature, when available.

\section{Standard patients}

\section{Definition/description of patient category}

Low-risk patients with a Model for End-Stage Liver Disease (MELD) score $<25$ and no autoimmune disease, HCC, or history of renal dysfunction.

\section{Relevant supporting evidence from the literature}

Early $(30 \pm 5$ days post-transplant) or very early ( $\leq 10$ days post-transplant) mTOR inhibitor introduction to reduce exposure to CNI has been reported to improve estimated glomerular filtration rate (eGFR) by $8-12 \mathrm{~mL} / \mathrm{min} / 1.73 \mathrm{~m}^{2}$ at 1 year after transplantation [15]. In the H2304 study, which investigated de novo use of everolimus in 719 liver transplant recipients who were randomized at $30 \pm 5$ days post-transplant, the adjusted change in eGFR at 12 months was superior in the everolimus plus reduced tacrolimus group using the Modification of Diet in Renal Disease (four-variable) (MDRD4) formula (difference of $8.5 \mathrm{~mL} /$ $\min / 1.73 \mathrm{~m}^{2} ; p<0.001$ ) [11], and remained significantly superior at 24 months (difference of $6.7 \mathrm{~mL} / \mathrm{min} / 1.73 \mathrm{~m}^{2}$; $p=0.002$ ) [12] compared with the standard tacrolimusbased regimen. An extension of the H2304 study demonstrated significantly improved renal function preservation for up to 3-years post-transplantation in 282 liver transplant recipients receiving everolimus plus reduced tacrolimus, with mean eGFR decreasing from randomization to month 36 by $7.0 \pm 31.3 \mathrm{~mL} / \mathrm{min} / 1.73 \mathrm{~m}^{2}$ versus $15.5 \pm 22.7 \mathrm{~mL} /$ $\min / 1.73 \mathrm{~m}^{2}$ in the tacrolimus-control group $(p=0.005)$ [18].

In the PROTECT trial, in which 203 liver transplant patients were randomized at 4 weeks post-transplant to discontinue CNI therapy and start everolimus or to continue their current CNI-based regimen, an eGFR treatment difference of $7.8 \mathrm{~mL} / \mathrm{min}(p=0.021)$ in favor of everolimus was identified at 12 months after transplantation using the MDRD4 formula [19]. At month 35 after randomization, a $10.1 \mathrm{~mL} / \mathrm{min}$ benefit in the adjusted mean eGFR in favor of everolimus was identified using the Cockcroft-Gault formula ( $p=0.082$ vs. CNI), $9.4 \mathrm{~mL} / \mathrm{min} / 1.73 \mathrm{~m}^{2}(p=0.053)$ using the MDRD4 formula, and $9.5 \mathrm{~mL} / \mathrm{min} / 1.73 \mathrm{~m}^{2}(p=0.028)$ using the Nankivell formula [14].

A separate study, in which 78 liver transplant patients were randomized to early CNI withdrawal followed by everolimus as monotherapy (with the initial dose of everolimus started on day 10 after liver transplantation) or to cyclosporine, identified a significant improvement in renal function at 12 months after transplantation in patients treated with everolimus compared with cyclosporine (mean eGFR values [MDRD formula] were $87.6 \pm 26.1 \mathrm{~mL} / \mathrm{min}$ vs. $59.9 \pm 12.6 \mathrm{~mL} / \mathrm{min}$, respectively; $p<0.001$ ) [20]. 


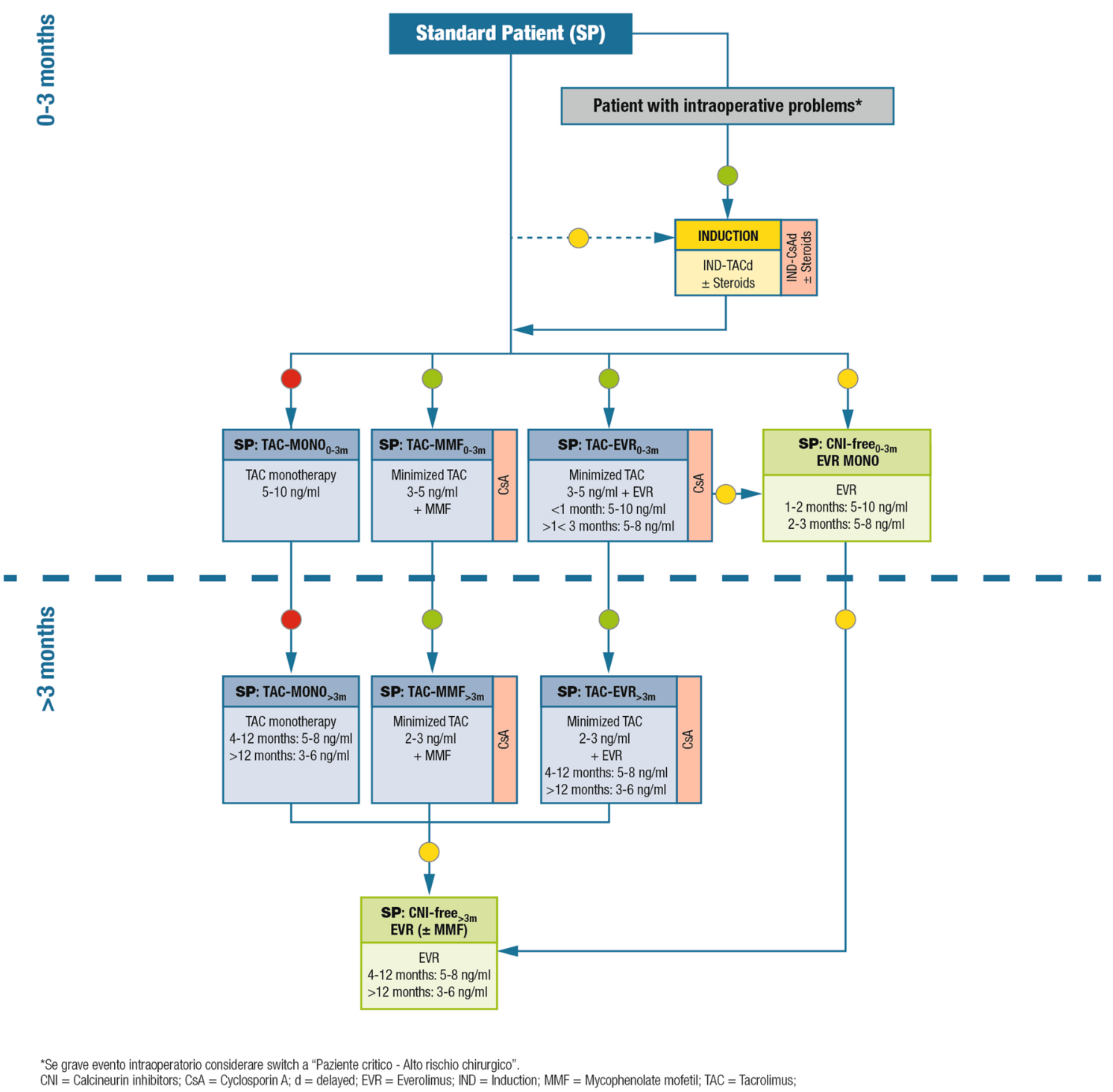

Fig. 1 Immunosuppression algorithm for standard patients. Key: green circle = recommended; yellow circle = caution advised; red circle = not recommended

Table 1 General remarks on the algorithms for immunosuppressive therapy in liver transplant recipients

\begin{tabular}{ll}
\hline & General remarks \\
\hline Blood levels & The indicated target values are not binding \\
Steroids & The orientation of the algorithm is steroid-free \\
CNI & As an alternative to tacrolimus, cyclosporine can be chosen \\
& (in particular in dysmetabolic patients with severe diabe- \\
& tes) \\
Induction & ATG can be chosen as an alternative to anti-IL-2R \\
$\begin{array}{l}\text { CNI-free schemes in which MMF is associ- } \\
\text { ated with everolimus }\end{array}$ & Evaluate a reduction of everolimus levels \\
MMF & As an alternative, the sodium salt form of MPA can be used
\end{tabular}

$A T G$ anti-thymocyte globulin, $C N I$ calcineurin inhibitor, $I L$ interleukin, $M M F$ mycophenolate mofetil, $M P A$ mycophenolic acid 
Table 2 Notes on the algorithms for immunosuppressive therapy in liver transplant recipients

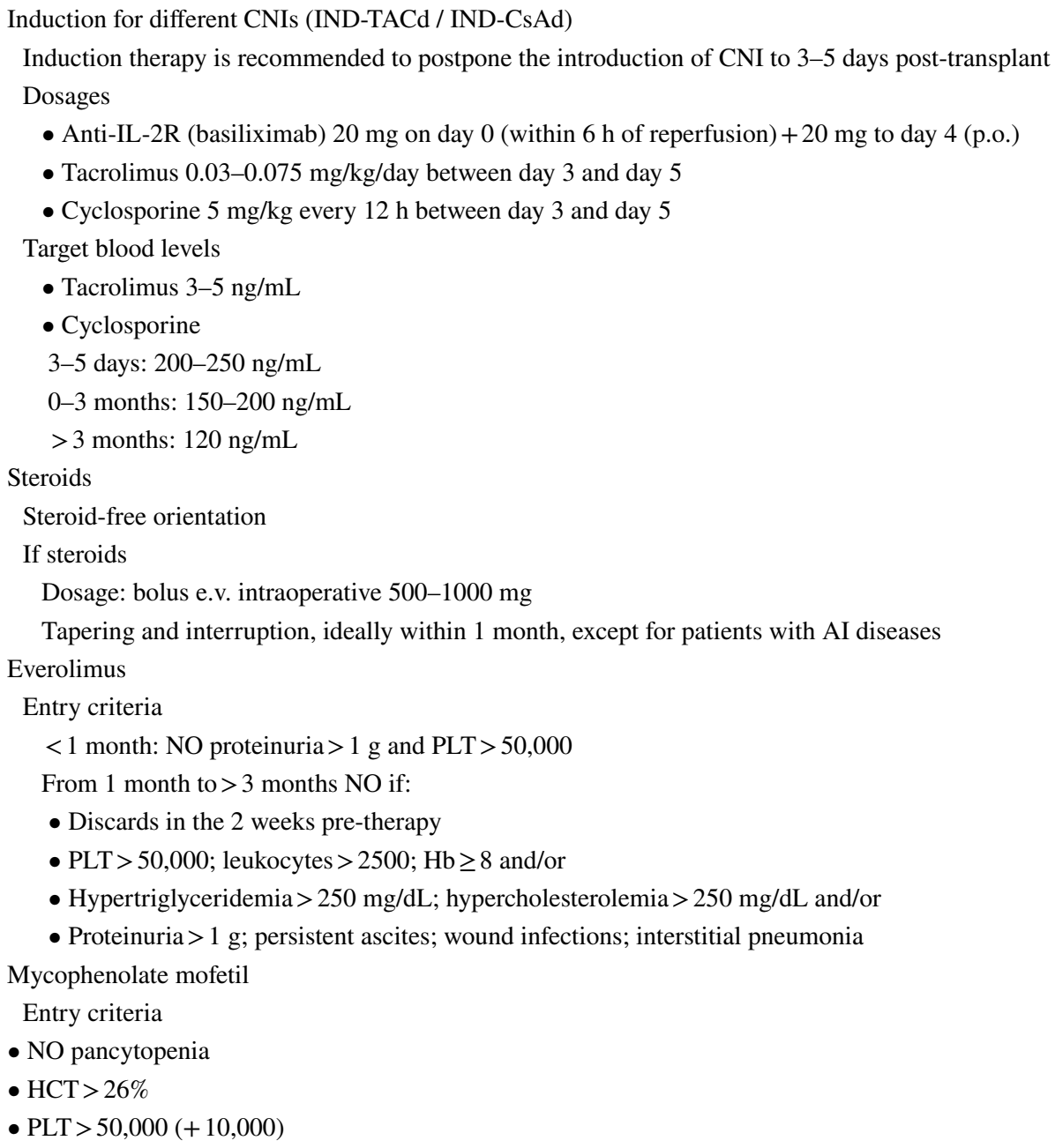

CNIs calcineurin inhibitors, $I L$ interleukin, $h$ hours, $H C T$ haematocrit, $H b$ hemoglobin, PLT platelets, p.o. by mouth
In addition, evidence from randomized studies and retrospective analyses shows that the improvement in renal function is limited if CNI-reducing strategies are postponed until renal function has deteriorated $\left(\right.$ eGFR $\left.<60 \mathrm{~mL} / \mathrm{min} / 1.73 \mathrm{~m}^{2}\right)$ [10, 21-23].

\section{Summary of recommendations and expert opinion}

In standard patients (Fig. 1), immunosuppression is based on CNIs, usually tacrolimus; cyclosporine can also be used. Tacrolimus monotherapy is not recommended. Early CNI reduction is feasible with the introduction of everolimus or mycophenolic acid derivatives, and may be further facilitated by the administration of induction agents (usually basiliximab for liver transplant recipients). Overall, a steroid-free approach is recommended.

\section{Critically ill patients}

\section{Definition/description of patient category}

Patients with one or more of the conditions listed in Fig. 2, namely high MELD-Na (i.e., MELD-Na $>29$ or MELD$\mathrm{Na} 25-29$ with concomitant renal dysfunction/dialysis or chronic encephalopathy), national high urgency, acute gastroesophageal bleeding, renal dysfunction/dialysis, hepatorenal syndrome, sepsis, spontaneous bacterial peritonitis, colonization by multidrug-resistant organisms, portal thrombosis, metabolic syndrome or non-alcoholic steatohepatitis, thoracic ascites, urinary tract infection and/or ventilatory support and/or inotropic support, or high surgical risk are considered as high-risk patients. Frail patients, with sarcopenia as a distinctive characteristic, also belong to this category [24-27]. 


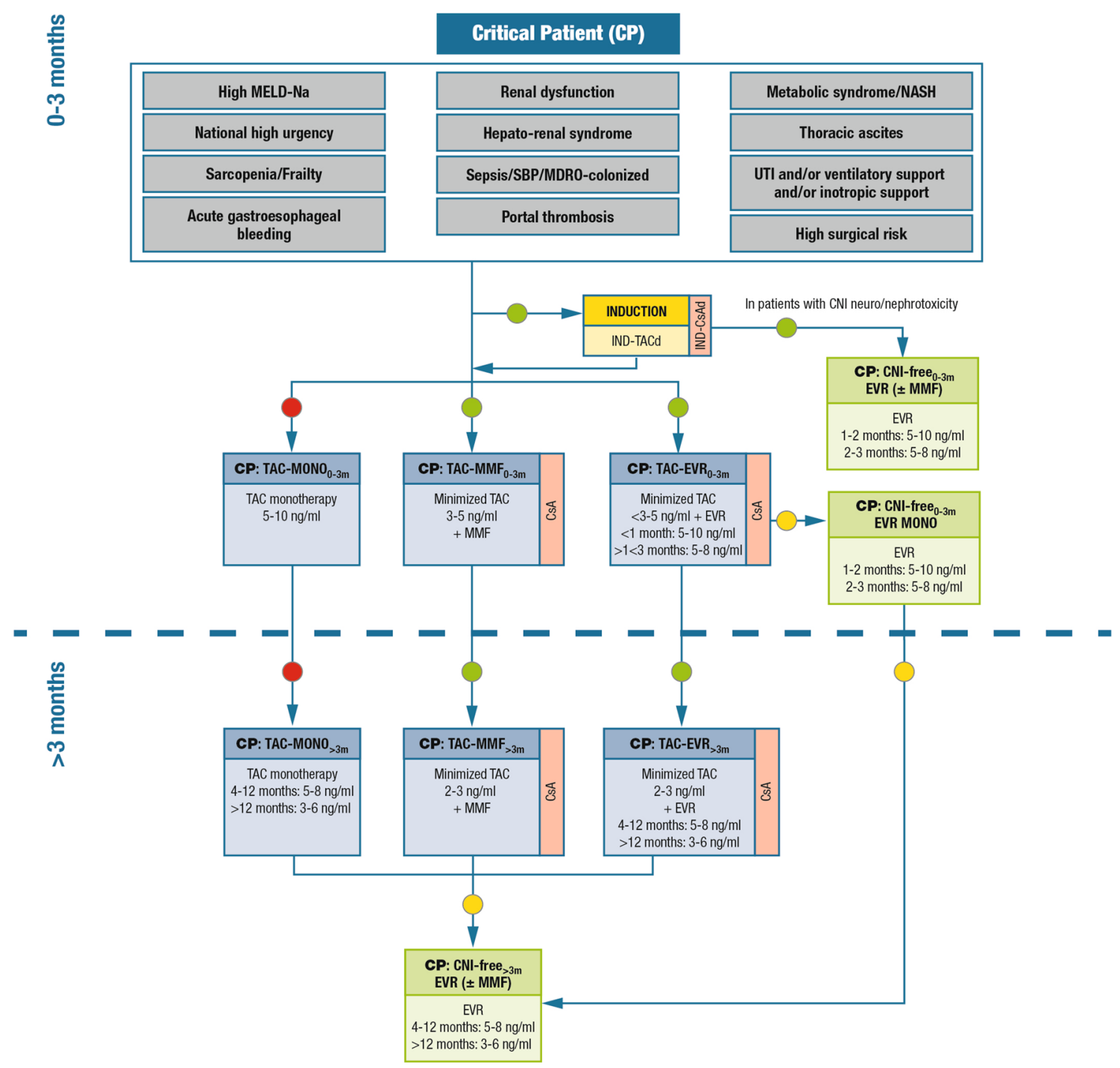

$\mathrm{CNI}=$ Calcineurin inhibitors; $\mathrm{CSA}=$ Cyclosporin A; $\mathrm{d}=$ delayed; EVR $=$ Everolimus; IND = Induction; MDRO = Multidrug-resistant organism; MELD-Na $=$ Model for End-stage Liver Disease - Sodium; MMF = mycophenolate mofetil; NASH = Non alcoholic steatohepatitis; SBP = Spontaneous bacterial peritonitis; TAC = Tacrolimus; UTI = Urinary tract infection;

Fig. 2 Immunosuppression algorithm for critically ill patients. Key: green circle = recommended; yellow circle = caution advised; red circle = not recommended

\section{Relevant supporting evidence from the literature}

Studies in this patient category are lacking, as most trials enroll low-risk patients (e.g., patients with no renal impairment).

\section{Summary of recommendations and expert opinion}

Induction therapy (basiliximab) is recommended in these patients to delay the introduction of CNI to 3-5 days posttransplantation. In patients with elevated MELD-Na (>29), the use of induction therapy should be carefully considered; CNI should be initiated at lower doses as compared to standard patients. CNI monotherapy is not recommended, and cyclosporine is a possible alternative to tacrolimus in particular in patients with dysmetabolic abnormalities and decompensated diabetes. For CNI reduction, the available options include mycophenolic acid derivatives and everolimus. CNI discontinuation can be considered for patients with signs of CNI-induced neuro-nephrotoxicity. Everolimus is particularly indicated in patients at risk of renal dysfunction or with renal dysfunction confirmed by eGFR assessments; among these patients, those with diabetes or metabolic disease may particularly benefit from everolimus, as this mTOR inhibitor has cardiovascular protective effects beside nephroprotective properties. Hyperlipidemia 
and hypertriglyceridemia, if present, can be treated before or after the introduction of everolimus. Proteinuria requires accurate renal function work-up and can be treated with appropriate strategies.

\section{Patients with a specific etiology}

\section{Definition/description of patient category}

In this group, we included less frequent or emerging indications to liver transplantation in Italy, as follows: patients with polycystic liver disease (PLD) with or without kidney involvement, patients necessitating combined liver-kidney transplantation, patients with autoimmune liver disease (AILD), patients with oncological indications to transplantation as neuroendocrine tumors (NET), metastatic colorectal cancer (metaCRC), or patients with cholangiocarcinoma (CCA) (Fig. 3).

\section{Relevant supporting evidence from the literature}

Two inherited disorders associated with PLD can be distinguished: isolated polycystic liver disease (PCLD) and autosomal dominant polycystic kidney disease (ADPKD). Although PCLD is not associated with excess mortality

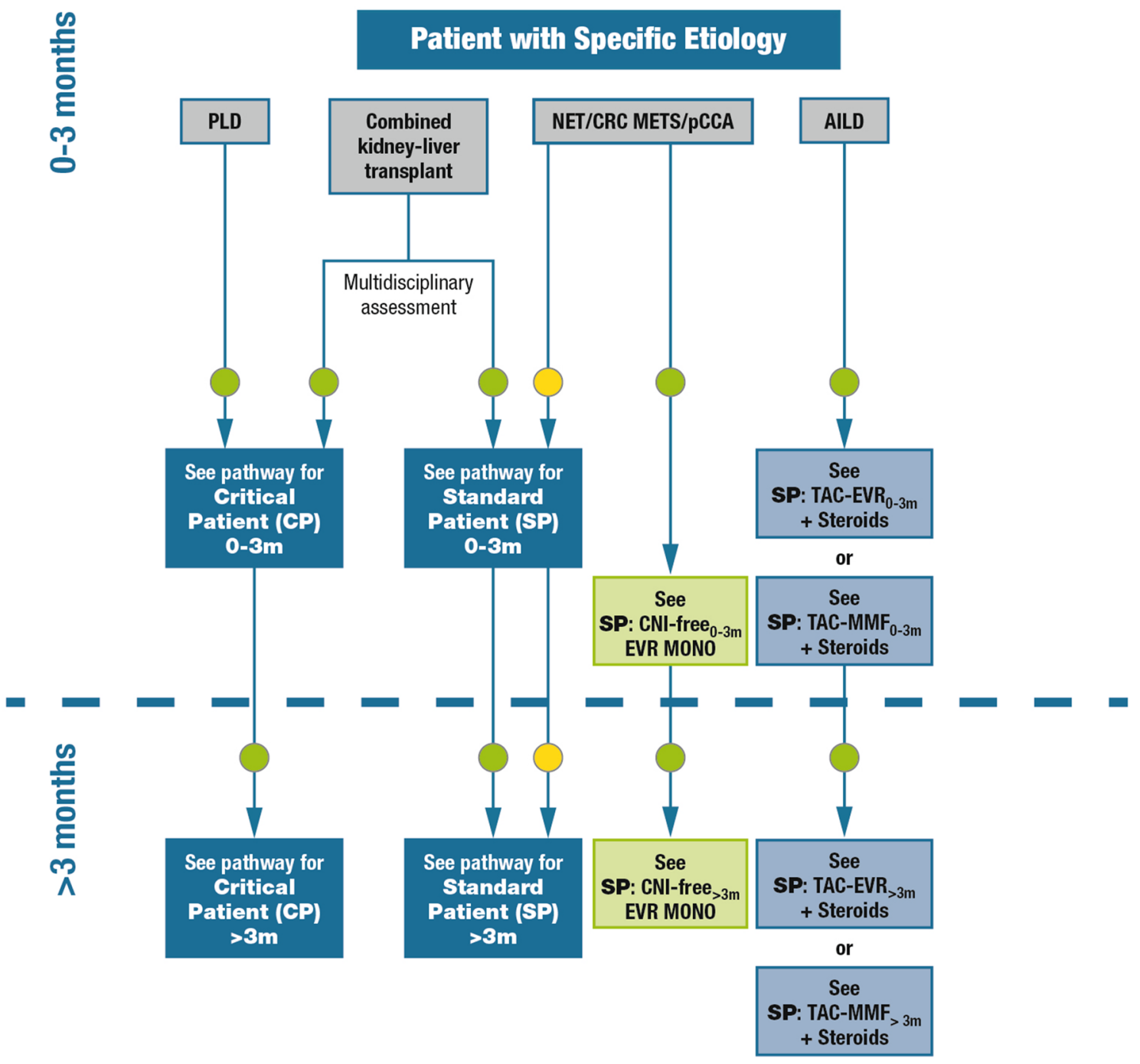

AlLD = Autoimmune liver disease; $\mathrm{CNI}=$ Calcineurin inhibitors; $\mathrm{CRC} \mathrm{METS} \mathrm{=} \mathrm{Colorectal} \mathrm{cancer} \mathrm{metastases;} \mathrm{EVR}=$ Everolimus; $\mathrm{MMF}=$ Mycophenolate mofetil; NET = Neuroendocrine tumors; PCCA $=$ Perihilar cholangiocarcinoma; PLD = Polycystic liver disease TAC = Tacrolimus

Fig. 3 Immunosuppression algorithm for patients with a specific etiology. Key: green circle = recommended; yellow circle =caution advised 
when left untreated, the quality of life supports the choice of liver transplantation as a therapeutic option [28, 29].

Combined liver-kidney transplantation should be considered in patients with severe renal impairment or on renal replacement therapy [30, 31].

Liver transplantation for NET liver metastasis may be a viable treatment option in selected patients as 5-year posttransplant overall survival rates of 47-97\% and disease-free survival rates of $32-87 \%$ have been reported [32-34]. Survival outcomes after liver transplantation are similar for liver transplantation performed in "ideal" candidates and for those with HCC [33]. However, as recurrence rates remain higher, immunosuppressive treatment must be appropriate to the oncological status.

For patients with metaCRC, the role of liver transplantation requires further elucidation and definition by ongoing clinical trials and real-life practice [35]. Nonetheless, a Norwegian study of 21 patients with nonresectable colorectal liver metastases demonstrated a 5-year survival rate of $60 \%$ after liver transplantation, exceeding outcomes reported for chemotherapy [36]. In colorectal cancer patients with nonresectable liver-only metastases, the 5-year overall survival rate was $83 \%$ after liver transplantation, highlighting the importance of improved patient selection criteria for optimal survival rates [37].

CCA, the second most common liver cancer, is associated with a very poor prognosis [38]. Liver transplantation protocols for CCA show low survival rates, given that tumor size $(>2 \mathrm{~cm})$ and multi-nodularity are risk factors for tumor recurrence and worse outcomes when compared with similar HCCs [39]. Nevertheless, clinically acceptable results were obtained in selected patients who underwent neoadjuvant chemoradiotherapy followed by liver transplantation [40]. In such cases, the immunosuppressive strategy must balance the risk of recurrence. There is also some experimental and clinical evidence on the antiproliferative effect of mTOR inhibitors in CCA-tumor cells [41] and in clinical studies [42].

\section{Summary of recommendations and expert opinion}

Patients with isolated PCLD can be referred to the critical patient path, due to complex surgery. Patients with ADPKD and renal impairment and/or in renal replacement therapy are considered for simultaneous or delayed combined liverkidney transplantation $[43,44]$. In this case, the immunosuppressive therapy is directed towards the critical patient path or towards the standard case path depending on the clinical conditions and after a careful multidisciplinary evaluation $[30,31]$.

Everolimus-facilitated CNI reduction should be implemented as early as possible, to avoid further damage to the kidney. In patients with oncological issues, the early introduction of mTOR inhibitors should be considered, and their use is also recommended in the case of post-transplant recurrence [45]. This could apply also to patients with additional risk factors for post-transplant de novo malignancy development (i.e. alcohol-related liver disease).

AILD should be treated with tacrolimus and corticosteroids, adjusting the latter based on efficacy and side effects; everolimus or mycophenolates can also be added. Cyclosporine can be used instead of tacrolimus [46], mostly in case of relapse.

\section{Patients with hepatocellular carcinoma}

\section{Definition/description of patient category}

Patients undergoing liver transplantation due to HCC.

\section{Relevant supporting evidence from the literature}

Antiproliferative properties of mTOR inhibitors and data from the literature support the use of this drug class in HCC patients. A large body of preclinical and clinical evidence has shown that mTOR inhibitors can limit HCC recurrence and progression in liver transplant recipients [47-49]. Sirolimus-based immunosuppression was beneficial after liver transplantation with significantly higher patient survival post-transplantation for $\mathrm{HCC}$ in a multivariate analysis of 2491 patients (hazard ratio $0.53,95 \%$ confidence interval [CI] $0.31-0.92 ; p \leq 0.05)$ [48]. mTOR inhibitors were also associated with significantly lower rates of $\mathrm{HCC}$ recurrence after liver transplantation when compared with CNIs (8\% vs. $13.8 \% ; p<0.001)$ in a systematic review of 3666 HCC liver transplant recipients [47]. However, significant benefits of mTOR inhibitors were only observed in patients within Milan criteria (i.e., low-risk patients) in a prospective, randomized, phase 3 clinical trial of sirolimus-based immunosuppression in $525 \mathrm{HCC}$ liver transplant recipients [50]. Moreover, HCC recurrence-free and overall survival benefits of mTOR inhibitors were apparent for up to 3-5 years post-transplantation, but were not sustained thereafter. An analysis of the US Scientific Registry of Transplant Recipients suggested beneficial, albeit not statistically significant, outcomes in terms of lower rates of HCC recurrence and cancer-specific mortality in recipients of liver transplantations for HCC receiving sirolimus-based immunosuppression, however, effects appeared to be modified by patient age at transplantation, with better outcomes identified for transplant recipients $>55$ years of age than those $\leq 55$ years [51].

For patients with recurrent HCC post-transplantation, evidence from retrospective analyses and case reports suggests that mTOR inhibitors should be introduced to slow disease progression due to their antiproliferative activity [52-54]. The addition of everolimus to sorafenib in a 46-year 
old male who experienced $\mathrm{HCC}$ recurrence 11 years after liver transplantation led to an approximately 50\% reduction in tumor size after 3-months of treatment, with further reductions of tumor size after 8 months [52]. In a retrospective cohort study of 31 patients with recurrent HCC after liver transplantation, the combined use of mTOR inhibitor (everolimus or sirolimus) and sorafenib identified a median overall survival of 19.3 months after initiation of the combined treatment, with a median time to disease progression of 6.77 months [54]. In a retrospective analysis of 7 liver transplantation recipients with HCC recurrence who were switched to everolimus plus sorafenib, 5 patients were alive after a median follow-up of 6.5 months (interquartile range [IQR] 14 months), 4 patients with tumor progression; the median time to progression was 3.5 (IQR 12) months [53].

Compared with CNI-based immunosuppression, mTORinhibitor-based immunosuppression significantly increased recurrence-free-survival at both 1- and 3-years post-transplantation (risk ratio [RR] 1.09 and 1.1, respectively) in a systematic review and meta-analysis of 23 studies on the survival and recurrence of $\mathrm{HCC}$ in liver transplant recipients [55]. In addition, treatment with mTOR inhibitors conferred a significant survival advantage in overall survival at 1-, 3- and 5-years post-transplant (RR: 1.07, 1.1, and 1.18, respectively), and a significantly lower rate of $\mathrm{HCC}$ recurrence (RR: 0.67) compared with conventional CNI-based immunosuppression.

\section{Summary of recommendations and expert opinion}

In all HCC patients (regardless of their risk of relapse), strategies to facilitate CNI reduction should be implemented to limit CNI-related renal toxicities and the impact of CNI exposure on cancer recurrence. Steroid-free immunosuppressive schedules are preferred (Fig. 4).

\section{Patients with de novo malignancies}

\section{Definition/description of patient category}

Liver transplant recipients who develop de novo malignancies any time following transplantation, including extrahepatic solid tumors, non-melanoma skin cancer, and lymphoproliferative diseases.

\section{Relevant supporting evidence from the literature}

A relatively large body of evidence from studies in heart, kidney, and liver transplant recipients suggests that the use of mTOR inhibitors leads to a reduced incidence of de novo malignancies after solid organ transplantation $[15,56$, 57]. The incidence of de novo malignancy within 963 days post-transplant was significantly lower in patients receiving sirolimus/everolimus compared with cyclosporine/tacrolimus $(0.60 \%$ vs. $1.81 \% ; p<0.001)$ in a retrospective analysis of 33,249 kidney transplant recipients, with the risk of developing any de novo malignancy reduced by $60 \%$ with sirolimus/everolimus immunosuppression (relative risk 0.4, 95\% CI 0.24-0.64; $p=0.0002$ ) [56]. A retrospective cohort study of 7217 kidney transplant recipients demonstrated a $46 \%$ significantly reduced risk of de novo malignancies in recipients treated with $\mathrm{mTOR}$ inhibitors compared with those never treated $(p<0.05)$ [57].

Further evidence supports the use of mTOR inhibitors in patients with de novo malignancies after liver transplantation [58-60]. In 6 liver transplantation patients who converted from CNI to everolimus due to de novo malignancies, which appeared 3-4 years post-transplantation, $83 \%(n=5)$ of patients remained disease-free after a mean follow-up time of $10 \pm 9$ months; lung carcinoma recurred at 12-months following conversion in 1 patient [58]. From a total of 4 liver transplantation recipients who were converted to sirolimus-based immunosuppression due to the development of aggressive de novo malignancies, 3 patients who converted within 90-120 days after surgical removal of the malignancy remained tumor-free at $12-33$ months post-conversion; 1 patient, who switched to sirolimus at 18 months after a mastectomy for breast cancer, died due to its recurrence [59].

In this setting, mTOR inhibitors may be used either as monotherapy or combined with reduced-exposure CNI, depending on individual risk factors (i.e., time post-transplantation, transplantation indication, age at transplantation, etc.) $[15,47,48,58-60]$.

\section{Summary of recommendations and expert opinion}

CNI reduction or discontinuation is recommended (Fig. 5). Given its antiproliferative properties, everolimus should be administered as soon as possible. mTOR inhibitor-based immunosuppression is warranted in all liver transplant recipients at high risk of de novo malignancies (i.e., human herpes virus-8-positive patients; patients with alcoholic cirrhosis; patients with concurrent inflammatory bowel disease; recipients of grafts from donors at risk of transmission of malignancies; Epstein Barr virus-DNA positivity after transplantation) [15].

\section{Acute rejection of the liver graft}

Based on the available evidence and experience of the scientific board members, the current guidelines were focused more on the mitigation of immunosuppression-related adverse events than on the treatment of acute rejection of the liver graft. For the management of acute rejection episodes, the scientific board invites the use of the International Liver 
Fig. 4 Immunosuppression algorithm for patients with hepatocellular carcinoma. Key: green circle $=$ recommended; yellow circle $=$ caution advised

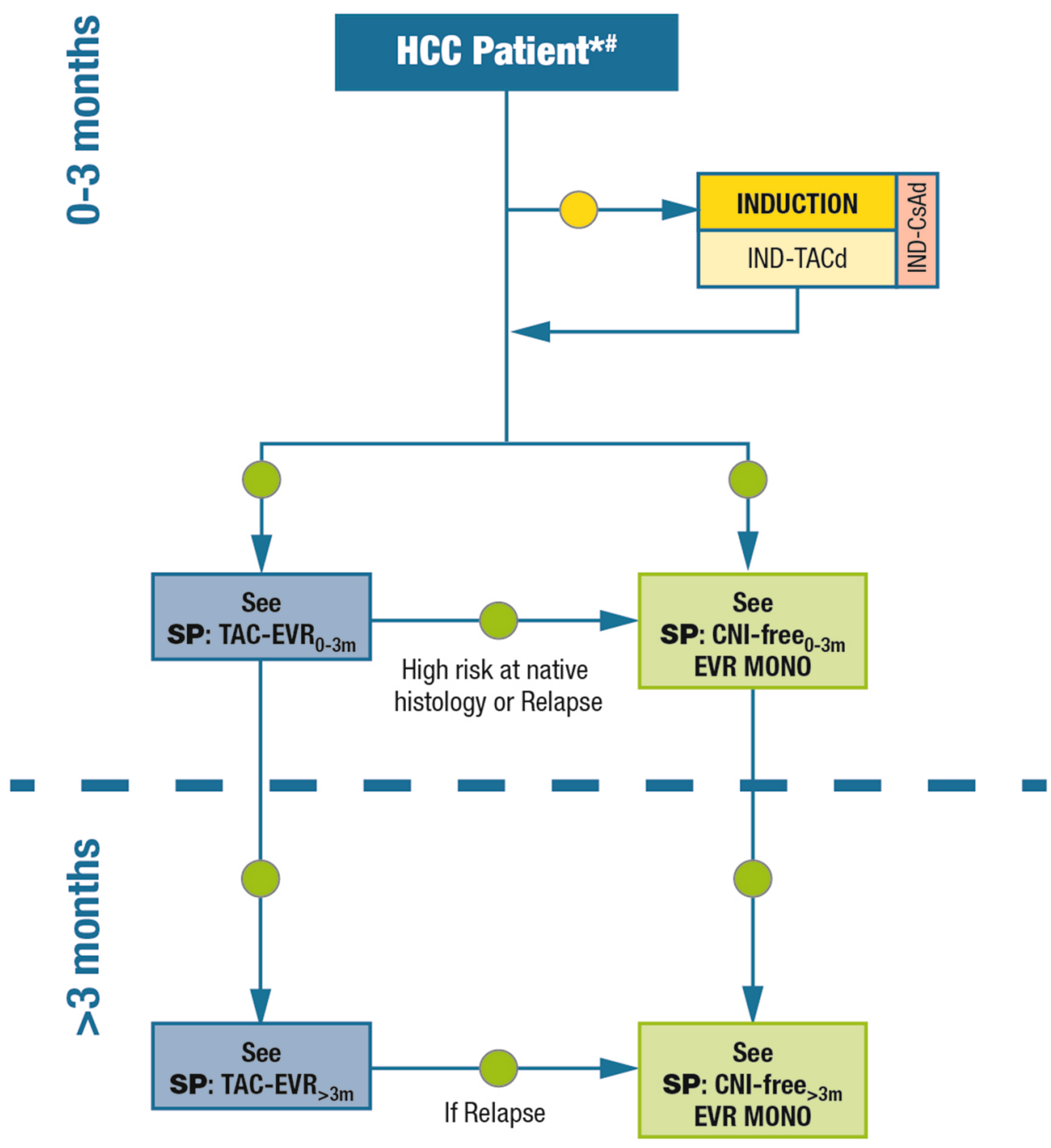

*This regimen is also suggested in patients with other primary or secondary hepatic malignancies. \#For both high risk and low risk settings the literature reports advantages of minimizing/eliminating $\mathrm{CNI}$ and the early use of mTORi.

$\mathrm{CNI}=$ Calcineurin inhibitors; $\mathrm{Cs} A=$ Cyclosporin $\mathrm{A} ; \mathrm{d}=$ delayed; EVR = Everolimus;

$\mathrm{HCC}=$ Hepatocellular carcinoma; IND = Induction;

$\mathrm{mTORi}=$ Mammalian target of rapamycin inhibitors; TAC $=$ Tacrolimus
Transplantation Society consensus guidelines on immunosuppression in liver transplant recipients [61].

\section{Discussion}

Although liver transplantation is well consolidated in Italy and elsewhere, a large amount of heterogeneity is apparent in the immunosuppression protocols used by different transplant centers. This is partly due to the observation that acute rejection of the liver graft does not affect graft or patient survival in adult liver transplantation, whilst focus is currently on the mitigation of immunosuppression-related adverse events [15]. As well as providing guidance to transplant physicians in the implementation of current recommendations for immunosuppressive therapy, the project undertaken in 2017 by the Italian Working Group aimed at standardizing immunosuppression regimens used in clinical practice with adult, ABO-compatible recipients. By dividing the population of liver transplant recipients into five distinct categories and by designing specific protocols for each category, the evidence- and consensus-based algorithms developed within the Italian Working Group, and presented here, may contribute to 
Fig. 5 Immunosuppression algorithm for patients with de novo malignancies. Key: green circle $=$ recommended; yellow circle $=$ caution advised

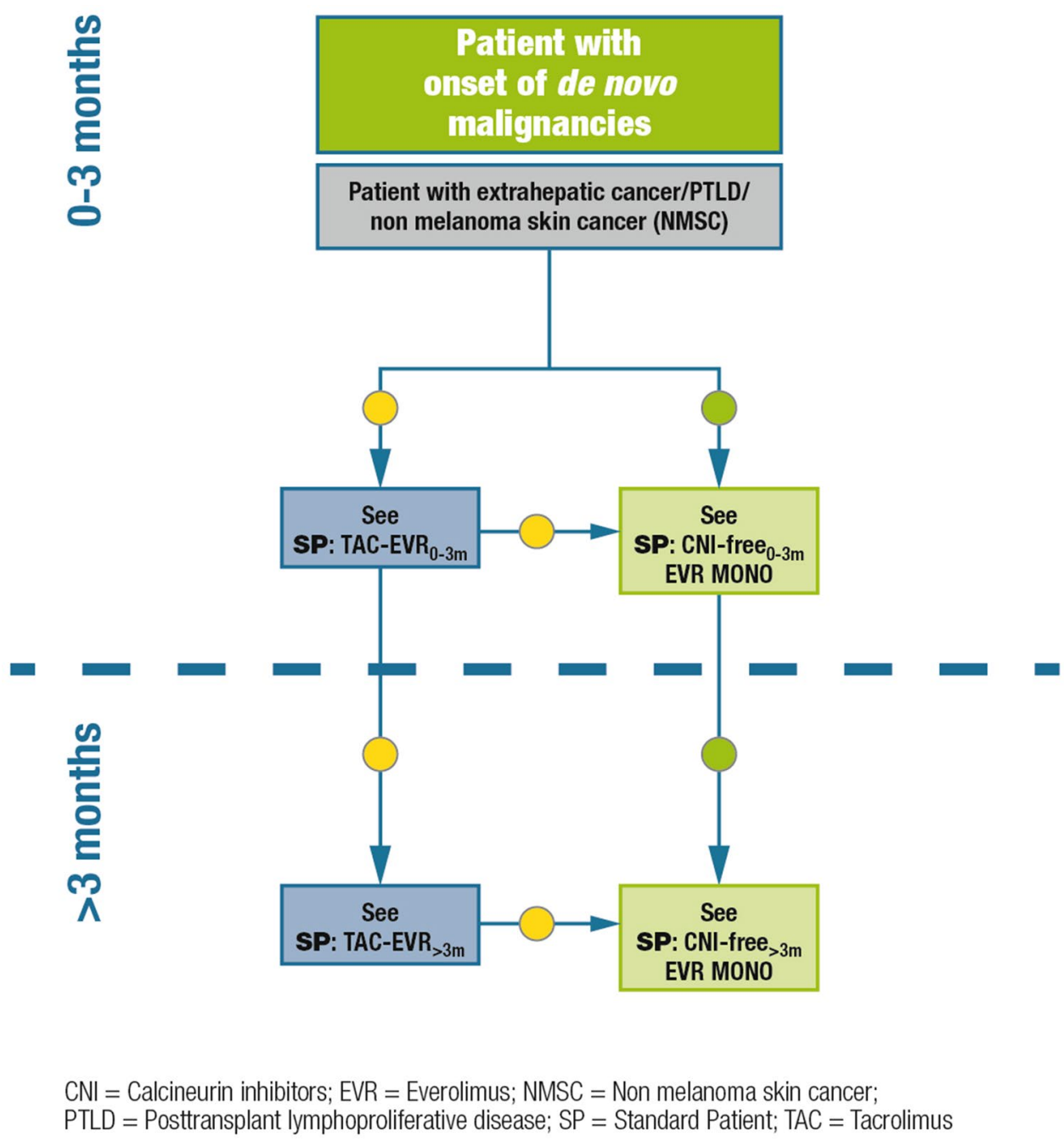

simplify, personalize, and optimize immunosuppression in clinical practice.

It is important to monitor renal function with adequate tools (i.e., eGFR) across all categories of liver transplant recipients. However, there remain a number of issues that are still controversial/unresolved including the management of proteinuria, and the need for additional evidence for the management of critically ill patients. The role and relevance of mTOR inhibitors in the prevention of HCC recurrence are; not definitively ascertained and need more scientific indepth work. Evidence supporting the use of mTOR inhibitors in patients with HCC recurrence is also very limited.

\section{Conclusion}

In conclusion, the choice of the immunosuppressive regimen in liver transplantation should take into account a composite of clinical variables, including primary disease, patient status at transplantation, type of surgery, early post-operative events, and the expected complications associated with longterm use of CNI, namely acute and chronic renal failure and de novo malignancies. Strategies for the prevention or limitation of CNI-related adverse events should be implemented as early as possible following transplantation, according to patient clinical status. At present, the most effective nephroprotective strategies include the reduction of CNI exposure facilitated by the early introduction of everolimus, or mycophenolate mofetil if everolimus is not indicated.

Acknowledgements Medical writing assistance was provided by Lorenza Lanini $(\mathrm{PhD})$ and Melanie Gatt $(\mathrm{PhD})$, independent medical writers, on behalf of Springer Healthcare.

Funding Novartis provided financial support for medical writing assistance.

\section{Compliance with ethical standards}

Conflict of interest Umberto Cillo declares that he has no conflict of interest. Luciano De Carlis declares that he has no conflict of inter- 
est. Massimo Del Gaudio declares that he has no conflict of interest. Paolo De Simone served as an advisory board member for Novartis, Astellas and Chiesi. Stefano Fagiuoli Advisory Board and Speaker's Bureau for Abbvie, Gilead Sciences, MSD, Novartis, Astellas, Bayer, Kedrion, Intercept. Francesco Lupo served as advisory board member for Novartis, Astellas, Biotest, Chiesi. Giuseppe Tisone declares that he has no conflict of interest. Riccardo Volpes declares that he has no conflict of interest.

Ethical approval This article does not contain any studies with human or animal subjects.

Open Access This article is licensed under a Creative Commons Attribution 4.0 International License, which permits use, sharing, adaptation, distribution and reproduction in any medium or format, as long as you give appropriate credit to the original author(s) and the source, provide a link to the Creative Commons licence, and indicate if changes were made. The images or other third party material in this article are included in the article's Creative Commons licence, unless indicated otherwise in a credit line to the material. If material is not included in the article's Creative Commons licence and your intended use is not permitted by statutory regulation or exceeds the permitted use, you will need to obtain permission directly from the copyright holder. To view a copy of this licence, visit http://creativecommons.org/licenses/by/4.0/.

\section{Appendix A}

\section{Names and affiliations of clinicians from Italian liver transplant centers who took part in the consensus panel}

Alfonso Avolio, Policlinico Universitario A. Gemelli-Roma

Davide Bitetto, Azienda Ospedaliero Universitaria di Udine-Udine

Patrizia Boccagni, Università di Padova-Padova

Amedeo Carraro, Azienda Ospedaliera Universitaria Integrata-Verona

Antonino Castellaneta, Azienda Ospedaliera Consorziale Policlinico di Bari-Bari

Michele Colledan, Azienda Socio Sanitaria Territoriale Papa Giovanni XXIII-Bergamo

Nicola De Maria, Azienda Ospedaliero Universitaria Policlinico di Modena-Modena

Fabrizio Di Benedetto, Azienda Ospedaliero Universitaria Policlinico di Modena-Modena

Alfonso Galeota Lanza, Azienda Ospedaliera Antonio Cardarelli-Napoli

Ivan Gardini, Presidente dell'associazione di pazienti EpaC onlus

Nicola Guglielmo, Polo Ospedaliero San Camillo-Forlanini-Spallanzani-Roma

Federica Invernizzi, Policlinico di Milano-Milano

Andrea Laurenzi, Polo Ospedaliero San Camillo-Forlanini-Spallanzani-Roma
Gioacchino Leandro, Specialista in Gastroenterologia, Epatologia e Metodologia della ricerca

Ilaria Lenci, Policlinico Ospedaliero Universitario Tor Vergata-Roma

Dario Lorenzin, Azienda Ospedaliero Universitaria di Udine-Udine

Laura Mameli, Azienda Ospedaliera G. Brotzu-Cagliari

Tommaso Maria Manzia, Policlinico Ospedaliero Universitario Tor Vergata-Roma

Anna Mariani, Ospedale Niguarda Ca' Granda-Milano

Gianluca Mennini, Azienda Policlinico Umberto

I-Roma

Stefano Mirabella, Azienda Ospedaliera Città della

Salute e della Scienza-Torino

Maria Cristina Morelli, Policlinico S. Orsola-Malpighi-Bologna

Daniele Nicolini, Ospedali Riuniti di Ancona-Ancona

Stefania Petruccelli, Azienda Ospedaliero Universitaria

Policlinico di Modena-Modena

Enrico Regalia, Istituto Nazionale dei Tumori di Milano-Milano

Paolo Reggiani, Policlinico di Milano-Milano

Stefania Roselli, Azienda Ospedaliera Consorziale Policlinico di Bari-Bari

Fausto Zamboni, Azienda Ospedaliera G. Brotzu-Cagliari

\section{References}

1. Nashan B. mTOR Inhibition and clinical transplantation: liver. Transplantation. 2018;102:S19-S26.

2. Gonwa TA, Mai ML, Melton LB, Hays SR, Goldstein RM, Levy MF, et al. End-stage renal disease (ESRD) after orthotopic liver transplantation (OLTX) using calcineurin-based immunotherapy: risk of development and treatment. Transplantation. 2001;72:1934-9.

3. Hojo M, Morimoto T, Maluccio M, Asano T, Morimoto K, Lagman M, et al. Cyclosporine induces cancer progression by a cellautonomous mechanism. Nature. 1999;397:530-4.

4. Ojo AO, Held PJ, Port FK, Wolfe RA, Leichtman AB, Young $\mathrm{EW}$, et al. Chronic renal failure after transplantation of a nonrenal organ. N Engl J Med. 2003;349:931-40.

5. Burra P, Rodriguez-Castro KI. Neoplastic disease after liver transplantation: focus on de novo neoplasms. World J Gastroenterol. 2015;21:8753-68.

6. Jain A, Marcos A, Reyes J, Mazariagos G, Kashyap R, Eghtesad B, et al. Tacrolimus for primary liver transplantation: 12 to 15 years actual follow-up with safety profile. Transplant Proc. 2005;37:1207-10.

7. Sharma P, Welch K, Eikstadt R, Marrero JA, Fontana RJ, Lok AS. Renal outcomes after liver transplantation in the model for end-stage liver disease era. Liver Transpl. 2009;15:1142-8.

8. Pham PT, Pham PC, Wilkinson AH. Management of renal dysfunction in the liver transplant recipient. Curr Opin Organ Transplant. 2009;14:231-9. 
9. Pillai AA, Levitsky J. Overview of immunosuppression in liver transplantation. World J Gastroenterol. 2009;15:4225-333.

10. De Simone P, Metselaar HJ, Fischer L, Dumortier J, Boudjema $\mathrm{K}$, Hardwigsen J, et al. Conversion from a calcineurin inhibitor to everolimus therapy in maintenance liver transplant recipients: a prospective, randomized, multicenter trial. Liver Transpl. 2009;15:1262-9.

11. De Simone P, Nevens F, De Carlis L, Metselaar HJ, Beckebaum S, Saliba F, et al. Everolimus with reduced tacrolimus improves renal function in de novo liver transplant recipients: a randomized controlled trial. Am J Transplant. 2012;12:3008-200.

12. Saliba F, De Simone P, Nevens F, De Carlis L, Metselaar HJ, Beckebaum S, et al. Renal function at two years in liver transplant patients receiving everolimus: results of a randomized, multicenter study. Am J Transplant. 2013;13:1734-45.

13. Saliba F, Dharancy S, Lorho R, Conti F, Radenne S, Neau-Cransac $\mathrm{M}$, et al. Conversion to everolimus in maintenance liver transplant patients: a multicenter, retrospective analysis. Liver Transpl. 2011;17:905-13.

14. Sterneck M, Kaiser GM, Heyne N, Richter N, Rauchfuss F, Pascher A, et al. Everolimus and early calcineurin inhibitor withdrawal: 3-year results from a randomized trial in liver transplantation. Am J Transplant. 2014;14:701-10.

15. De Simone P, Fagiuoli S, Cescon M, De Carlis L, Tisone G, Volpes R, et al. Use of everolimus in liver transplantation: recommendations from a working group. Transplantation. 2017;101:239-51

16. Fagiuoli S, Burra P, Salizzoni M, Cescon M, De Carlis L, Di Costanzo GG, et al. Renal protection strategies are poorly implemented after liver transplantation: the 12-month results of the Italian national study SURF. Abstract BOS433. Transpl Int. 2017;30(Suppl 2):289.

17. Cillo U, Burra P, Mazzaferro V, Belli L, Pinna AD, Spada M, et al. A multistep, consensus-based approach to organ allocation in liver transplantation: toward a "Blended Principle Model". Am J Transplant. 2015;15:2552-611.

18. Fischer L, Saliba F, Kaiser GM, De Carlis L, Metselaar HJ, De Simone P, et al. Three-year outcomes in de novo liver transplant patients receiving everolimus with reduced tacrolimus: followup results from a randomized, multicenter study. Transplantation. 2015;99:1455-62.

19. Fischer L, Klempnauer J, Beckebaum S, Metselaar HJ, Neuhaus $\mathrm{P}$, Schemmer P, et al. A randomized, controlled study to assess the conversion from calcineurin-inhibitors to everolimus after liver transplantation-PROTECT. Am J Transplant. 2012;12:1855-65.

20. Masetti M, Montalti R, Rompianesi G, Codeluppi M, Gerring R, Romano A, et al. Early withdrawal of calcineurin inhibitors and everolimus monotherapy in de novo liver transplant recipients preserves renal function. Am J Transplant. 2010;10:2252-62.

21. Abdelmalek MF, Humar A, Stickel F, Andreone P, Pascher A, Barroso E, et al. Sirolimus conversion regimen versus continued calcineurin inhibitors in liver allograft recipients: a randomized trial. Am J Transplant. 2012;12:694-705.

22. Castroagudin JF, Molina E, Romero R, Otero E, Tome S, Varo E. Improvement of renal function after the switch from a calcineurin inhibitor to everolimus in liver transplant recipients with chronic renal dysfunction. Liver Transpl. 2009;15:1792-7.

23. De Simone P, Precisi A, Petruccelli S, Balzano E, Carrai P, Catalano G, et al. The impact of everolimus on renal function in maintenance liver transplantation. Transplant Proc. 2009;41:1300-2.

24. Carey EJ, Lai JC, Wang CW, Dasarathy S, Lobach I, MontanoLoza AJ, et al. A multicenter study to define sarcopenia in patients with end-stage liver disease. Liver Transpl. 2017;23:625-33.
25. Fried LP, Tangen CM, Walston J, Newman AB, Hirsch C, Gottdiener J, et al. Frailty in older adults: evidence for a phenotype. $\mathrm{J}$ Gerontol A Biol Sci Med Sci. 2001;56:M146-156.

26. Guralnik JM, Simonsick EM, Ferrucci L, Glynn RJ, Berkman LF, Blazer DG, et al. A short physical performance battery assessing lower extremity function: association with self-reported disability and prediction of mortality and nursing home admission. J Gerontol. 1994;49:M85-94.

27. Martin L, Birdsell L, Macdonald N, Reiman T, Clandinin MT, McCargar LJ, et al. Cancer cachexia in the age of obesity: skeletal muscle depletion is a powerful prognostic factor, independent of body mass index. J Clin Oncol. 2013;31:1539-47.

28. Drenth JP, Chrispijn M, Nagorney DM, Kamath PS, Torres VE. Medical and surgical treatment options for polycystic liver disease. Hepatology. 2010;52:2223-30.

29. Gevers TJ, Drenth JP. Diagnosis and management of polycystic liver disease. Nat Rev Gastroenterol Hepatol. 2013;10:101-8.

30. Pita A, Kaur N, Emamaullee J, Lo M, Nguyen B, Sabour A, et al. Outcomes of liver transplantation in patients on renal replacement therapy: considerations for simultaneous liver kidney transplantation versus safety net. Transplant Direct. 2019;5:e490.

31. Sharma P. Liver-kidney: indications, patient selection, and allocation policy. Clin Liver Dis (Hoboken). 2019;13:165-9.

32. Gedaly R, Daily MF, Davenport D, McHugh PP, Koch A, Angulo $\mathrm{P}$, et al. Liver transplantation for the treatment of liver metastases from neuroendocrine tumors: an analysis of the UNOS database. Arch Surg. 2011;146:953-8.

33. Lim C, Lahat E, Osseis M, Sotirov D, Salloum C, Azoulay D. Liver transplantation for neuroendocrine tumors: what have we learned? Semin Liver Dis. 2018;38:351-6.

34. Mazzaferro V, Sposito C, Coppa J, Miceli R, Bhoori S, Bongini $\mathrm{M}$, et al. The long-term benefit of liver transplantation for hepatic metastases from neuroendocrine tumors. Am J Transplant. 2016;16:2892-902.

35. Chotai $P$, Matsuoka L. Reassessing the role of liver transplantation for patients with metastatic colorectal cancer to the liver. Curr Opin Organ Transplant. 2019;24:118-20.

36. Hagness M, Foss A, Line PD, Scholz T, Jorgensen PF, Fosby B, et al. Liver transplantation for nonresectable liver metastases from colorectal cancer. Ann Surg. 2013;257:800-6.

37. Dueland S, Syversveen T, Solheim JM, Solberg S, Grut H, Bjornbeth BA, et al. Survival following liver transplantation for patients with nonresectable liver-only colorectal metastases. Ann Surg. 2020;271:212-8.

38. Goldaracena N, Gorgen A, Sapisochin G. Current status of liver transplantation for cholangiocarcinoma. Liver Transpl. 2018;24:294-303.

39. Sapisochin G, de Lope CR, Gastaca M, de Urbina JO, LopezAndujar R, Palacios F, et al. Intrahepatic cholangiocarcinoma or mixed hepatocellular-cholangiocarcinoma in patients undergoing liver transplantation: a Spanish matched cohort multicenter study. Ann Surg. 2014;259:944-52.

40. Zaborowski A, Heneghan HM, Fiore B, Stafford A, Gallagher $\mathrm{T}$, Geoghegan J, et al. Neoadjuvant chemoradiotherapy and liver transplantation for unresectable hilar cholangiocarcinoma: the Irish experience of the Mayo Protocol. Transplantation. 2020;104(10):2097-104.

41. Heits N, Heinze T, Bernsmeier A, Kerber J, Hauser C, Becker T, et al. Influence of mTOR-inhibitors and mycophenolic acid on human cholangiocellular carcinoma and cancer associated fibroblasts. BMC Cancer. 2016;16:322.

42. Wu CE, Chen MH, Yeh CN. mTOR inhibitors in advanced biliary tract cancers. Int J Mol Sci. 2019;20:500.

43. Coquillard C, Berger J, Daily M, Shah M, Mei X, Marti F, et al. Combined liver-kidney transplantation for polycystic liver and 
kidney disease: analysis from the united network for organ sharing dataset. Liver Int. 2016;36:1018-25.

44. Lauterio A, De Carlis R, Di Sandro S, Buscemi V, Andorno E, De Carlis L. Delayed kidney transplantation in combined liver-kidney transplantation for polycystic liver and kidney disease. Transpl Int. 2019;32:1336-8.

45. Rousseau B, Guillemin A, Duvoux C, Neuzillet C, Tlemsani C, Compagnon $\mathrm{P}$, et al. Optimal oncologic management and mTOR inhibitor introduction are safe and improve survival in kidney and liver allograft recipients with de novo carcinoma. Int J Cancer. 2019;144:886-96.

46. Montano-Loza AJ, Hansen BE, Corpechot C, Roccarina D, Thorburn D, Trivedi $P$, et al. Factors associated with recurrence of primary biliary cholangitis after liver transplantation and effects on graft and patient survival. Gastroenterology. 2019;156(96-107):e101.

47. Cholongitas E, Mamou C, Rodriguez-Castro KI, Burra P. Mammalian target of rapamycin inhibitors are associated with lower rates of hepatocellular carcinoma recurrence after liver transplantation: a systematic review. Transpl Int. 2014;27:1039-49.

48. Toso C, Merani S, Bigam DL, Shapiro AM, Kneteman NM. Sirolimus-based immunosuppression is associated with increased survival after liver transplantation for hepatocellular carcinoma. Hepatology. 2010;51:1237-43.

49. Treiber G. mTOR inhibitors for hepatocellular cancer: a forwardmoving target. Expert Rev Anticancer Ther. 2009;9:247-61.

50. Geissler EK, Schnitzbauer AA, Zulke C, Lamby PE, Proneth A, Duvoux C, et al. Sirolimus use in liver transplant recipients with hepatocellular carcinoma: a randomized, multicenter, open-label phase 3 trial. Transplantation. 2016;100:116-25.

51. Yanik EL, Chinnakotla S, Gustafson SK, Snyder JJ, Israni AK, Segev DL, et al. Effects of maintenance immunosuppression with sirolimus after liver transplant for hepatocellular carcinoma. Liver Transpl. 2016;22:627-34.

52. Bhoori S, Toffanin S, Sposito C, Germini A, Pellegrinelli A, Lampis A, et al. Personalized molecular targeted therapy in advanced, recurrent hepatocellular carcinoma after liver transplantation: a proof of principle. J Hepatol. 2010;52:771-5.

53. De Simone P, Crocetti L, Pezzati D, Bargellini I, Ghinolfi D, Carrai $\mathrm{P}$, et al. Efficacy and safety of combination therapy with everolimus and sorafenib for recurrence of hepatocellular carcinoma after liver transplantation. Transplant Proc. 2014;46:241-4.

54. Gomez-Martin C, Bustamante J, Castroagudin JF, Salcedo M, Garralda E, Testillano M, et al. Efficacy and safety of sorafenib in combination with mammalian target of rapamycin inhibitors for recurrent hepatocellular carcinoma after liver transplantation. Liver Transpl. 2012;18:45-52.

55. Grigg SE, Sarri GL, Gow PJ, Yeomans ND. Systematic review with meta-analysis: sirolimus- or everolimus-based immunosuppression following liver transplantation for hepatocellular carcinoma. Aliment Pharmacol Ther. 2019;49:1260-73.

56. Kauffman HM, Cherikh WS, Cheng Y, Hanto DW, Kahan BD. Maintenance immunosuppression with target-of-rapamycin inhibitors is associated with a reduced incidence of de novo malignancies. Transplantation. 2005;80:883-9.

57. Piselli P, Serraino D, Segoloni GP, Sandrini S, Piredda GB, Scolari MP, et al. Risk of de novo cancers after transplantation: results from a cohort of 7217 kidney transplant recipients, Italy 1997-2009. Eur J Cancer. 2013;49:336-44.

58. Bilbao I, Sapisochin G, Dopazo C, Lazaro JL, Pou L, Castells $\mathrm{L}$, et al. Indications and management of everolimus after liver transplantation. Transplant Proc. 2009;41:2172-6.

59. Vivarelli M, Dazzi A, Cucchetti A, Gasbarrini A, Zanello M, Di Gioia P, et al. Sirolimus in liver transplant recipients: a large single-center experience. Transplant Proc. 2010;42:2579-84.

60. Jimenez-Romero C, Manrique A, Marques E, Calvo J, Sesma AG, Cambra F, et al. Switching to sirolimus monotherapy for de novo tumors after liver transplantation. A preliminary experience. Hepatogastroenterology. 2011;58:115-21.

61. Charlton M, Levitsky J, Aqel B, O’Grady J, Hemibach J, Rinella $\mathrm{M}$, et al. International liver transplantation society consensus statement on immunosuppression in liver transplant recipients. Transplantation. 2018;102:727-43.

Publisher's Note Springer Nature remains neutral with regard to jurisdictional claims in published maps and institutional affiliations. 